\title{
Research on Confinement Loss Characteristics of Crystal Fibers
}

\author{
Yuqin Yao ${ }^{1, a}$, Shiyu $\mathbf{H u}^{2}$ \\ ${ }^{1}$ Chengdu University of Information Technology, Chengdu, 610225, China \\ ${ }^{2}$ Sichuan Meiguhe hydropower development company, LTD Chengdu, 611130 , China \\ ${ }^{a}$ Email: yyq@cuit.edu.cn
}

Keywords: Photonic crystal, loss, fiber communication

\begin{abstract}
Since the born of the photonic crystal fiber, because of the excellent characteristics which is superior to the traditional fiber, it has become research hotspots in the field of optical fiber communication, optical sensing and optical devices in recent years. However, the loss of fiber has been restricting an obstacle in light signal transmission. The loss of traditional quartz fiber was about $0.15 \mathrm{~dB} / \mathrm{km}$, which means the signal power will be half after $20 \mathrm{~km}$. In this paper, by increasing the perfectly matched layer, according to the imaginary part of the effective refractive, we calculate the confinement loss of two polarization direction, analyzed different parameters, especially the influence of changing number of air hole layers and air hole pitch on the confinement loss. The experimental results show that, the confinement loss can be controlled within level $10^{-7}$ $(\mathrm{dB} / \mathrm{m})$, it has great reference value for high speed and long distance optical communication.
\end{abstract}

\section{The geometric structure of fiber}

In our design, the geometry structure of photonic crystal fiber is shown in Figure 1.1; the core shape has two types: rectangular and elliptical, we mainly study the elliptical core. In the outer square cladding we add the perfect matching layer with thickness of 3 me. The cladding layer and the matching layer refractive index are assumed as $1.45^{[1]}$. The way we study the confinement loss characteristics mainly focus on the changing of layer range, in our paper, it embodies in the changing of air hole layers, the changing of size and distance of air holes.

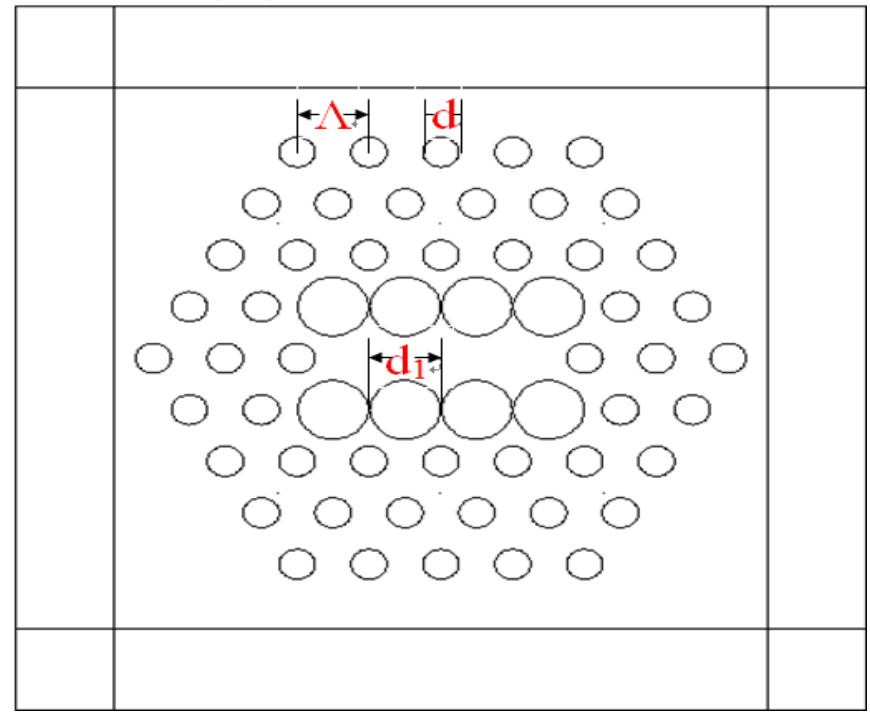

Figure 1.1 cross section of PCF 


\section{2 . The confinement loss characteristics of total internal reflection photonic crystal fiber}

The confinement loss a particular characteristic with crystal fiber, mainly because of the periodic structure (ideally) in cladding, it causes the resonance effect between the transmission power in the core and cladding structure, resulting in leakage of energy into the cladding, and then the signal gradually delays away in the transmission ${ }^{[2]}$. Now, in order to eliminate the resonance effect, there comes with the anti- resonating fiber. Mainly by filling high refractive index liquid into air hole, and it makes the refractive index of cladding higher than the core, this kind of fiber is called total solid fiber. The structure of fiber has great effect on confinement loss. In this paper, we first make analysis on single polarization single mould photonic crystal fiber, find the best fiber structure, and then analyze the effective of structure parameters on confinement loss ${ }^{[3]}$.

In real application, the limit and vanish of mode field is a very important; in order to measure correctly and eliminate boundary effect, there comes with the idea of absorbing boundary with perfect matching layer ${ }^{[4]}$. Adding perfect matching layer on the outside of photonic crystal, when calculate the value of effective refractive index, there will exist one imaging part, we can calculate the value of confinement loss according to the formula:

$$
\text { conf.Loss }=\frac{40 \pi}{\ln (10) \lambda} \operatorname{Im}\left(n_{\text {eff }}\right)=8.686 k_{0} \operatorname{Im}\left(n_{\text {eff }}\right)\left[\frac{d B}{m}\right]
$$

In this formula, $k_{0}$ is the wave number in free space.

\section{The influence of wavelength on confinement loss}

As shown in figure 3.1, it shows the relationship of confinement loss and changing wavelength of $\mathrm{x}$ and $\mathrm{y}$ direction on the condition that $\mathrm{d}_{1} / \Lambda=8$, air hole distance $\Lambda=2.0 \mu \mathrm{m}$, air hole layer number $\mathrm{N}=5^{[5]}$. From the figure, we can find when wavelength is $1.1 \mu \mathrm{m}$, the confinement loss level on $\mathrm{x}$ and $\mathrm{y}$ direction is $10^{-4}$ and $10^{-3}$; but when wavelength is $1.8 \mu \mathrm{m}$, the confinement loss level on $\mathrm{x}$ and $\mathrm{y}$ direction is $10^{-0}$ and $10^{-2}$. That is because with the increasing of wavelength, horizontal field outward expansion effect is enhancing (see figure 3.2) that cause more light-wave leakage from cladding. Through figure 3.1, we can also find when the wavelength ranging from 1.1 to $1.8 \mu \mathrm{m}$, the loss in $\mathrm{x}$ direction is one order lower than $\mathrm{y}$ direction that is because the fiber in this paper is realizing based on removing three air holes on s direction, it makes the size of air holes on $y$ polarization direction bigger. 


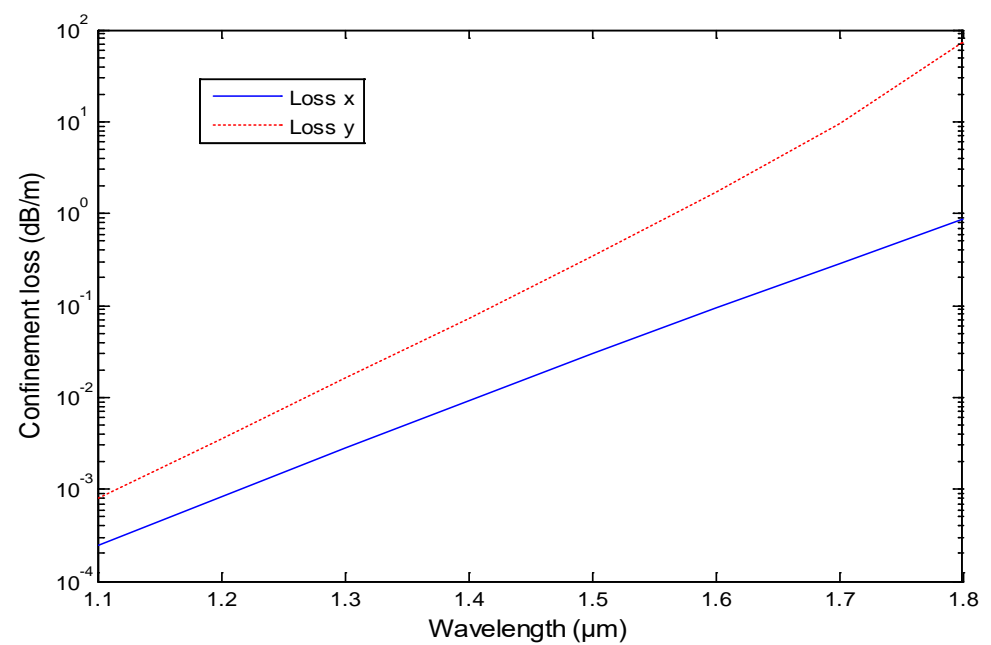

Figure 3.1 Dependence of confinement loss on the wavelength

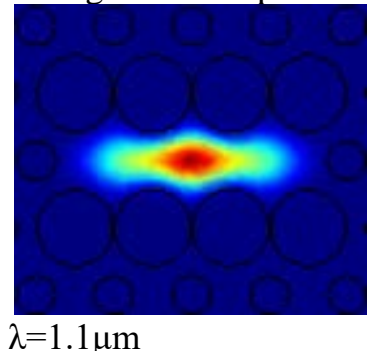

$\lambda=1.1 \mu \mathrm{m}$

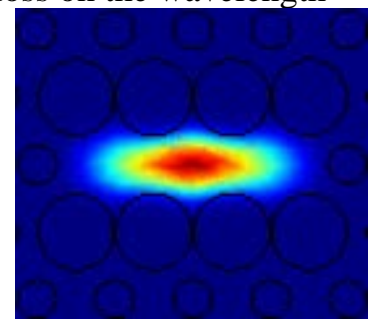

$\lambda=1.8 \mu \mathrm{m}$

Figure 3.2 modal field pattern for two proposed PCFs with $\lambda=1.1 \mu \mathrm{m}$ and $1.8 \mu \mathrm{m}$

\section{4 . The influence of air hole layer number on confinement loss}

On the condition that air hole distances $\Lambda=2.0 \mu \mathrm{m}, \mathrm{d} 1 / \Lambda=0.98$, air hole layer number are $4,5,6$ and 7 , the loss of $x$ direction along with the changing wavelength is shown in figure 4.1. From the figure, when wavelength is $1.1 \mu \mathrm{m}$, if the air hole layer increases one, the loss will reduce two orders; when wavelength is $1.8 \mu \mathrm{m}$, if the air hole layer increases one, the loss will reduce one order. According to it, we can preliminary obtain: with the increase of air hole number, confinement loss reduces fast. That is because the light can reach every cladding when transmitting, and part of the light exists leakage. The more the layer is, the leakage is weaker, the loss is lower. By comparing with other parameters, changing the number of layers is an important way to control the confinement loss. 


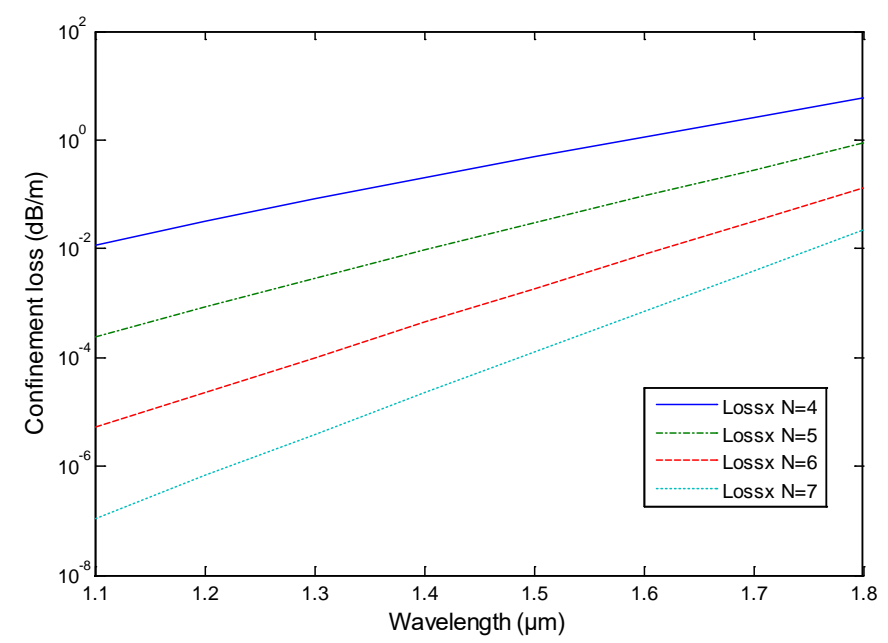

Figure 4.1 dependence of confinement loss on the number of air hole rings

\section{The influence of air hole distance on confinement loss}

On the condition that $\mathrm{d} / \Lambda=0.5, \mathrm{~d}_{1} / \Lambda=0.98$, air hole layer number is five, air hole distance are $1.8 \mu \mathrm{m}, 2.0 \mu \mathrm{m}, 2.2 \mu \mathrm{m}$. The confinement loss on $\mathrm{x}$ direction changes along with wavelength. As we can see in figure 5.1, with the distance increasing, the confinement loss appears decreasing trend. That is because when $d / \Lambda$ keeps the same value, if $\Lambda$ changes, it can change the rate of air which filled in the whole cladding, and then influence the confinement loss.

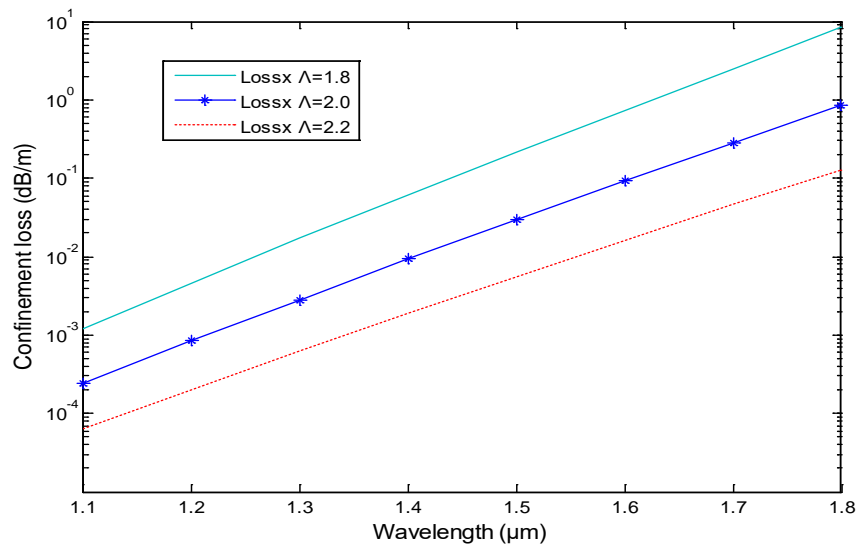

Figure 5.1 dependence of confinement loss on the air-holes with a pitch

\section{Conclusions}

In this paper, we first introduce the reason why total internal reflection photonic crystal fiber has confinement loss, point out several analysis theories about confinement loss of crystal fibers and the ways how to calculate the confinement loss. We mainly have a study on the influence of wavelength $\lambda$, the number of air hole layer $\mathrm{N}$ and air hole distance to confinement loss of PCF. We preliminary get the following conclusions: under the premise of one variable, if the wavelength within a fixed range, confinement loss will increase with the wavelength increases; reduce with the air hole layer number increases; reduce with the air hole distance increases. To the referring structure of fiber, changing the number of air hole layer and the distance between air holes are good ways to reduce 
confinement loss.

\section{References}

[1] Abdur M, Yoshinori N, Kazuya M, et al. Dispersion and confinement loss control in modified hexagonalphotonic crystal fibers. Optical Review. 2007

[2] S.Yang,Y. Zhang,X.Penget al. Theoretical study and experimental fabrication of high negative dispersion photonic crystal fiber with large area mode field. Optics Express. 2006

[3] Saitoh K,Koshiba M. Numerical modeling of photonic crystal fibers. Journal of Lightwave Technology. 2005

[4] D. Y. Tang,L. M. Zhao. "Generation of 47-fs pulses directly from an erbium-doped fiber laser". Optics Letters. 2007

[5] H. P. Uranus, H. Hoekstra."Modelling of microstructured waveguides using a finite-element-based vectorial mode solver with transparent boundary conditions," Optics Express. 2004 